\title{
A case of autosomal recessive hypercholesterolemia with a novel mutation in the LDLRAP1 gene
}

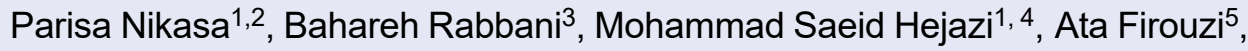 \\ Hossein Baharvand ${ }^{2,6}$, Mehdi Totonchi ${ }^{2}$, and Nejat Mahdieh ${ }^{3,5}$ \\ ${ }^{1}$ Department of Molecular Medicine, Faculty of Advanced Biomedical Sciences, Tabriz University of Medical \\ Sciences, Tabriz, Iran \\ ${ }^{2}$ Department of Stem Cells and Developmental Biology, Cell Science Research Center, Royan Institute for Stem Cell \\ Biology and Technology, ACECR, Tehran, Iran \\ ${ }^{3}$ Growth and Development Research Center, Tehran University of Medical Sciences, Tehran, Iran \\ ${ }^{4}$ Molecular Medicine Research Center, Biomedicine Institute, Tabriz University of Medical Sciences, Tabriz, Iran \\ ${ }^{5}$ Cardiogenetic Research Center, Rajaie Cardiovascular Medical and Research Center, Iran University of Medical \\ Sciences, Tehran, Iran \\ ${ }^{6}$ Department of Developmental Biology, School of Basic Sciences and Advanced Technologies in Biology, \\ University of Science and Culture, Tehran, Iran
}

Key words: autosomal recessive hypercholesterolemia, premature atherosclerotic cardiovascular disease, $L D L R A P 1$ gene, rosuvastatin, ezetimibe

\section{Introduction}

Familial hypercholesterolemia (FH, OMIM number \#143890), a life-threatening monogenic disorder characterized by high levels of low-density lipoprotein cholesterol (LDL-C), is classified into dominant and recessive types (1). The dominant form of $\mathrm{FH}$ may result from mutations in the $L D L R, A P O B$, and PCSK9 genes (2). However, mutations in the low-density lipoprotein receptor (LDLR) adaptor protein-1 (LDLRAP1) gene cause an autosomal recessive inheritance pattern of FH called autosomal recessive hypercholesterolemia (ARH, OMIM number \#603813). The LDLRAP1 protein, encoded by the $L D L R A P 1$ gene, is required for receptormediated endocytosis of LDL-C (2). ARH is a rare disorder with an estimated prevalence of less than 1 in a population of one million. This disease is considered a phenocopy of the most severe form of $\mathrm{FH}$, homozygous familial hypercholesterolemia (HoFH; OMIM number 143890). Hence, most ARH patients are clinically indiscernible from HoFH, which is caused by two defective $L D L R$ genes with an approximate prevalence of one individual per million (8). Considering that ARH patients may develop aggressive and premature atherosclerotic cardiovascular disease (ASCVD) due to hypercholesterolemia during early adulthood, lipidlowering therapy must be initiated during childhood (3). Early identification of ARH patients through genetic analysis of the proband and their relatives can provide prognosis and subsequently appropriate, timely treatment. In this report, we describe a novel variant, c. $649 \mathrm{G}>\mathrm{T}$, p.Glu217Ter, in the homozygous state in exon 7 of the $L D L R A P 1$ gene, causing severe ARH.

\section{Case Report}

The patient was a 20-yr-old woman from a small village in Ilam, Iran. When she was 10 yr old, the earliest clinical manifestations became apparent, and she was referred to a physician because of the existence of

Received: June 15, 2021 Accepted: July 18, 2021

Co-corresponding authors: Mehdi Totonchi, Ph.D., Department of Stem Cells and Developmental Biology, Cell Science Research Center, Royan Institute, Banihashem Sq., Banihashem St., Ressalat highway, Tehran 193954644, Iran

E-mail: m.totonchi@Royaninstitute.org

Nejat Mahdieh, Ph.D., Cardiogenetic Research Center, Rajaie Cardiovascular Medical and Research Center, Valiasr Street, Tehran 1995614331, Iran

E-mail: nmahdieh@gmail.com 
tendon xanthomas on her hands, elbows, and knees. The patient was aware of her severe hypercholesterolemia since she was 11 yr old, with an LDL-C concentration of $720 \mathrm{mg} / \mathrm{dL}$. Daily pharmacological treatment with 4 $\mathrm{g}$ cholestyramine was initiated. The patient reported that she had undergone subaortic web resection and aortoplasty due to uncontrolled hyperlipidemia at 13 yr of age. She was referred to our cardiovascular center at $17 \mathrm{yr}$ of age for the study of hypercholesterolemia. Bilateral corneal arcus, xanthomas, and xanthelasmas were present. The plasma lipid profile revealed severe hypercholesterolemia: total cholesterol (TC), $520 \mathrm{mg} /$ dL; low-density lipoprotein-cholesterol (LDL-C), 446 $\mathrm{mg} / \mathrm{dL}$; high-density lipoprotein-cholesterol (HDL-C), $57 \mathrm{mg} / \mathrm{dL}$; and triglyceride (TG), $93 \mathrm{mg} / \mathrm{dL}$. Secondary causes of hypercholesterolemia, including renal disease, diabetes mellitus, and thyroid disease, were ruled out. The liver enzyme levels were normal. Physical examination revealed a blood pressure of 110/70 $\mathrm{mmHg}$ and a body mass index (BMI) of $29 \mathrm{~kg} / \mathrm{m}^{2}$. Family history revealed that the parents were cousins and had given birth to four children (two males and two females), two of whom had passed away for unknown reasons. The lipid profile of the patient's mother (at the age of 44 yr) was in the normal range: TC, $198 \mathrm{mg} / \mathrm{dL}$; LDL-C,
$105 \mathrm{mg} / \mathrm{dL}$; HDL-C, $62 \mathrm{mg} / \mathrm{dL}$; and TG, $150 \mathrm{mg} / \mathrm{dL}$. The patient's father had died at the age of $42 \mathrm{yr}$ from coronary artery disease, and his lipid profile was unavailable (Fig. 1A). Genetic analysis revealed a homozygous mutation c. $649 \mathrm{G}>\mathrm{T}$ (p.Glu217Ter) in the LDLRAP1 gene of the patient. Based on these findings, the patient was diagnosed with $\mathrm{ARH}$. According to the results of coronary angiography, the patient was a candidate for coronary artery bypass grafting (CABG) and aortic valve replacement (AVR); however, the patient refused this surgery for personal reasons. Pharmacological treatment with rosuvastatin $(60 \mathrm{mg} / \mathrm{d})$ and ezetimibe (10 mg/d) was initiated. The treatment process and the consequent lipid responses are shown in Table 1. While undergoing this treatment, the cutaneous xanthomas decreased markedly, and a substantial reduction in plasma LDL-C was noted (from $402.5 \pm 31.1$ to 103.8 $\pm 26.02 \mathrm{mg} / \mathrm{dL})$. This reduction was associated with a markedly increase in alanine aminotransferase (from $16.25 \pm 6.05$ to $49.2 \pm 25.2 \mathrm{UI} / \mathrm{L}$ ). The changes in lipid levels obtained at baseline (mean of four determinations) and during cholesterol-lowering treatment (mean of monthly determinations) are shown in Table 1. After 3 years of treatment, despite using maximal recommended rosuvastatin and ezetimibe doses, the results of coronary
A

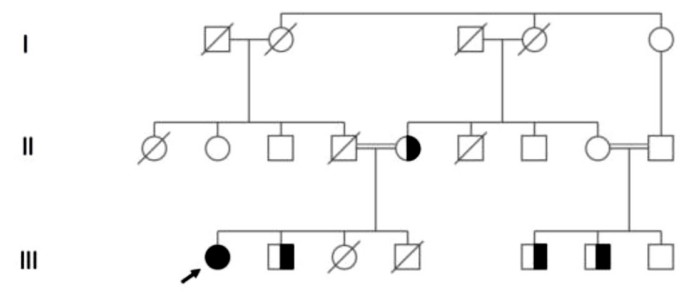

B

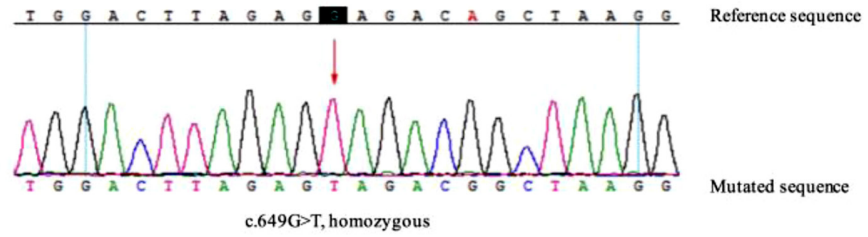

C

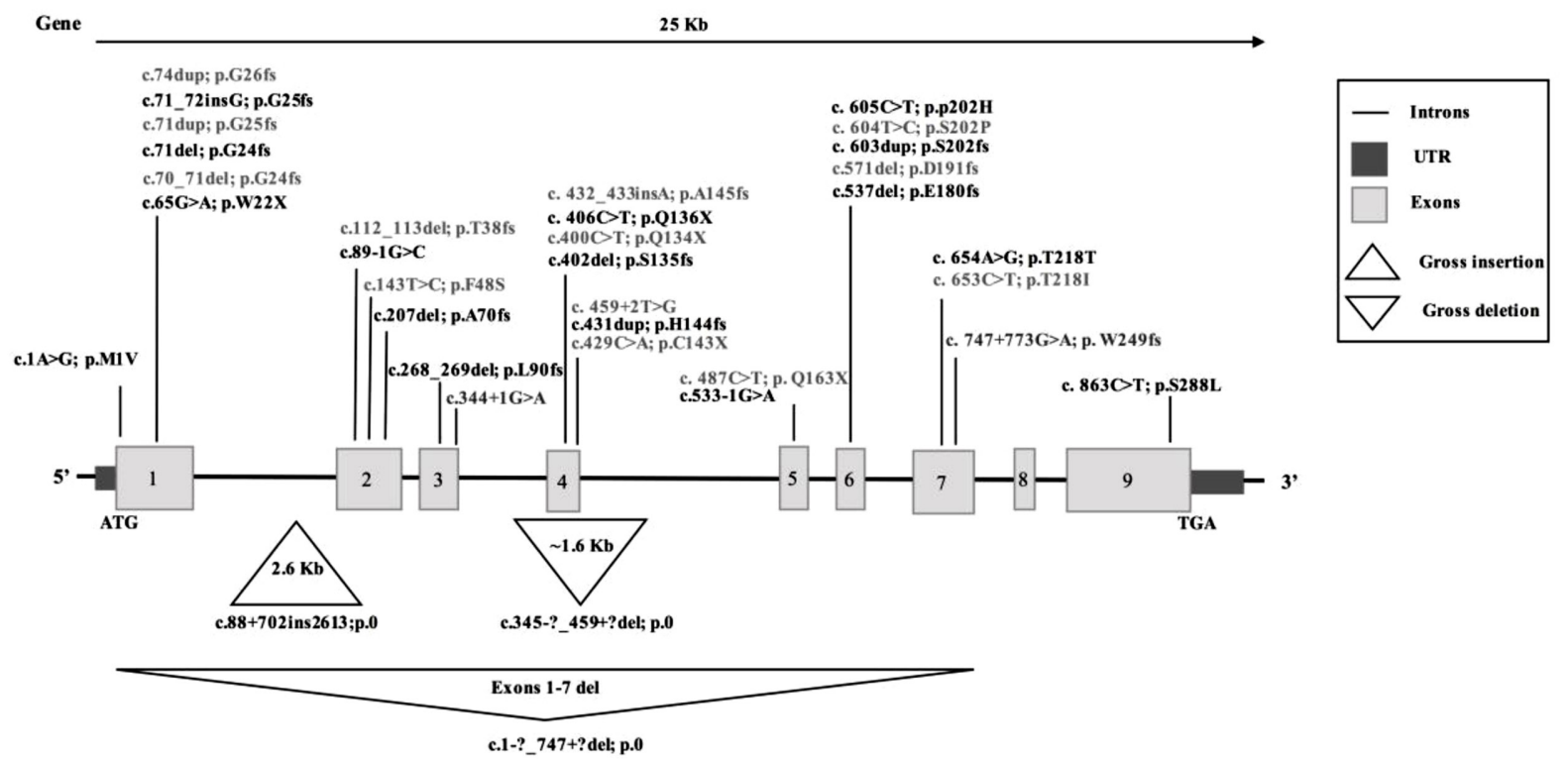

Fig. 1. A: Pedigree of the family with ARH. The proband indicated by the black arrow carries the novel mutation c.649G $>$ T in LDLRAP1. The mother and brother were heterozygotes for the same mutation. B: Chromatogram indicating the novel mutation in exon 7 of LDLRAP1 in the affected individual. C: Updated version of mutations in ARH patients determined to date. 
Table 1. Evolution of lipid profile of the patient before and during treatment with rosuvastatin $(60 \mathrm{mg} / \mathrm{d})$ plus ezetimibe $(10 \mathrm{mg} / \mathrm{d})$

\begin{tabular}{lcccc}
\hline Plasma parameter & Before drugs & R60 + E10 & Percent change & $\mathrm{P}^{*}$ \\
\hline TC mg/dL & $482.25 \pm 28.4$ & $169.7 \pm 32.1$ & -64.8 & $<0.0001$ \\
LDL-C mg/dL & $402.5 \pm 31.1$ & $103.8 \pm 26.02$ & -74.2 & $<0.001$ \\
HDL-C mg/dL & $61.5 \pm 5.4$ & $48.1 \pm 6.8$ & -21.8 & $<0.01$ \\
TG mg/dL & $81.5 \pm 13.6$ & $62.8 \pm 11.5$ & -22.9 & $<0.052$ \\
\hline
\end{tabular}

TC, total cholesterol; LDL-C, low-density lipoprotein cholesterol; HDL-C, high-density lipoprotein cholesterol; TG, triglycerides Values are mean \pm SD. * Comparisons were performed using Student's t-test for paired data.

angiography demonstrated that severe supravalvular aortic stenosis (SVAS) resulted in significant stenotic lesions of the coronary arteries and aortic valve. Hence, it was suggested that the patient should undergo CABG and AVR, but she refused to do so again.

\section{Mutational Analysis}

After obtaining informed consent from the proband's mother, we extracted genomic DNA from the peripheral blood samples of the proband when she was $17 \mathrm{yr}$ old. Gene panel-based next-generation sequencing (NGS) was performed to identify causal variant(s) in the known genes involved in $\mathrm{FH}$, including $L D L R$, LDLRAP1, $P C S K 9$, and $A P O B$ (4). Sanger sequencing was used to validate the presence of the new variant identified via NGS (Fig. 1B). A novel homozygous variant (c.649G>T) in the LDLRAP1 gene was detected in this patient. The same mutation was identified in LDLRAP1 in heterozygosity for her mother and living brother. The identified nonsense variant was absent in HGMD, dbSNP version 147, ClinVar databases, Iranome, and Exome Sequencing Project. This variant has not been found in the existing literature. The sequence variant was submitted to the ClinVar database (Accession number: VCV000981055.1; https://www.ncbi.nlm.nih. gov/clinvar/variation/981055/). c.649G $>$ T leads to the formation of a stop codon at amino acid residue 217 of the LDLRAP1 protein (p.Glu217Ter). The results of NGS analysis did not identify any pathogenic changes in $L D L R, P C S K 9$, and $A P O B$.

\section{Discussion}

$\mathrm{ARH}$ is a genetic disorder of lipid metabolism caused by disruptive mutations in both alleles of the LDLRAP1 gene (4). The $L D L R A P 1$ gene is $25-\mathrm{kb}$ long and contains nine exons. It is located on the short arm of chromosome 1 (1p36.11) and encodes the LDLRAP1 protein with 308 amino acids. According to the currently available information in the ClinVar database and literature review, 34 pathogenic variants in the coding sequence of LDLRAP1 have been reported (Fig. 1C) $(1,3,5,6)$. In this clinical report, we described a novel variant, c.649G $>\mathrm{T}$, p.Glu217Ter, in exon 7 of LDLRAP1. The patient is most likely homozygous for c.649G>T (p.Glu217Ter) in the
LDLRAP1 gene, since the parents are consanguineous. However, as the patient's father had died many years ago and we could not perform genetic analysis for him, the possibility of a large deletion, including exon 7 of the LDLRAP1 gene, cannot be excluded (5). In other words, the patient might be compound heterozygous for large deletions, including exon 7 and c.649G>T (p.Glu217Ter).

In silico analysis was performed using available software tools, including CADD, SIFT, and MutationTaster, to predict the pathogenicity of the variant. The results of the analysis predicted that this variant was damaging due to the generation of a premature stop codon in exon 7 of LDLRAP1. Furthermore, we recently determined the functional consequence of this mutation by disease modeling of the novel $L D L R A P 1$ variant c. $649 \mathrm{G}>\mathrm{T}$ in patient-specific induced pluripotent stem cell-derived hepatocyte-like cells (HLCs) (7). This study demonstrated that LDLuptake by HLCs was disrupted by this mutation. Hence, our findings confirmed the pathological effects of this mutation.

$\mathrm{HoFH}$, the most severe form of $\mathrm{FH}$, is considered a phenocopy of ARH. Therefore, most ARH patients are clinically indiscernible from HoFH. The risk of aortic valve stenosis (AVS) and ASCVD is similar between them. However, SVAS is a rare finding in ARH patients (6), which is typically observed in $\mathrm{HoFH}$.

The lipid-lowering response of $\mathrm{ARH}$ patients is much better than that of $\mathrm{HoFH}$ patients (8), specifically LDLR-negative patients. However, there is great variability in the LDL-C lowering response to statin treatment in $\mathrm{ARH}$ patients, ranging from $20 \%$ to $90 \%$ (6). In our ARH patient, a 74\% reduction in plasma LDL-C concentration was achieved through a high dose of rosuvastatin plus ezetimibe.

In spite of conventional therapies, the cardiovascular prognosis of ARH is poor (3). Despite treatment with a high dose of rosuvastatin plus ezetimibe, our patient presented with severe cardiovascular involvement, AVS, and SVAS at a very early age. Consequently, our findings highlight the importance of early identification of ARH patients via genetic analysis to improve prognosis and determine appropriate treatment.

Conflict of interests: The authors declare no conflicts of interest. 


\section{Acknowledgments}

We would like to thank the patient and her family for their willingness to participate in our research and their cooperation with us. This work was supported in part by a research grant to Dr. Mahdieh from Iran's National Science Foundation (98023504) and the Research Deputy of Iran University of Medical Sciences (98-3-37-16373).

\section{References}

1. Fellin R, Arca M, Zuliani G, Calandra S, Bertolini S. The history of Autosomal Recessive Hypercholesterolemia (ARH). From clinical observations to gene identification. Gene 2015;555: 23-32. [Medline] [CrossRef]

2. Mikhailova S, Ivanoshchuk D, Timoshchenko O, Shakhtshneider E. Genes potentially associated with familial hypercholesterolemia. Biomolecules 2019;9: 807. [Medline] [CrossRef]

3. D’Erasmo L, Di Costanzo A, Arca M. Autosomal recessive hypercholesterolemia: update for 2020. Curr Opin Lipidol 2020;31: 56-61. [Medline] [CrossRef]

4. Petrulioniene Z, Gargalskaite U, Mikstiene V, Norvilas R, Skiauteryte E, Utkus A. Autosomal recessive hypercholesterolemia: Case report. J Clin Lipidol 2019;13: 887-93. [Medline] [CrossRef]

5. Eden ER, Patel DD, Sun XM, Burden JJ, Themis M, Edwards M, et al. Restoration of LDL receptor function in cells from patients with autosomal recessive hypercholesterolemia by retroviral expression of ARH1. J Clin Invest 2002;110: 1695-702. [Medline] [CrossRef]

6. Vaverkova H, Tichy L, Karasek D, Freiberger T. A case of autosomal recessive hypercholesterolemia caused by a new variant in the LDL receptor adaptor protein 1 gene. J Clin Lipidol 2019;13: 405-10. [Medline] [CrossRef]

7. Nikasa P, Tricot T, Mahdieh N, Baharvand H, Totonchi M, Hejazi MS, et al. Patient-specific induced pluripotent stem cell-derived hepatocyte-like cells as a model to study autosomal recessive hypercholesterolemia. Stem Cells Dev 2021;30: 714-24. [Medline] [CrossRef]

8. Gidding SS, Champagne MA, de Ferranti SD, Defesche J, Ito MK, Knowles JW, et al. American Heart Association Atherosclerosis, Hypertension, and Obesity in Young Committee of Council on Cardiovascular Disease in Young, Council on Cardiovascular and Stroke Nursing, Council on Functional Genomics and Translational Biology, and Council on Lifestyle and Cardiometabolic Health. The agenda for familial hypercholesterolemia: a scientific statement from the American Heart Association. Circulation 2015;132: 2167-92. [Medline] [CrossRef] 